\title{
ON THE SPACE OF MATRICES OF GIVEN RANK
}

\author{
by M. C. CRABB and D. L. GONÇALVES
}

(Received 9th July 1987)

\section{Introduction}

Let $V$ and $W$ be finite dimensional real vector spaces, $k \geqq 0$ an integer. We write $L(V, W)$ for the space of all linear maps $V \rightarrow W$ and $L_{k}(V, W)$ for the subspace of maps with kernel of dimension $k$; in particular, $L_{0}(V, W)$ is the open subspace of injective linear maps. Thus $L_{k}\left(\mathbb{R}^{n}, \mathbb{R}^{n}\right)$ is the space of $n \times n$-matrices of rank $n-k$ in the title. We also need the notation $G_{k}(V)$ for the Grassmann manifold of $k$-dimensional subspaces of $V$.

In this note we shall investigate the homotopy theory of the smooth fibre bundle

$$
\pi: L_{k}(V, W) \rightarrow G_{k}(V)
$$

obtained by mapping an element of $L_{k}(V, W)$ to its kernel. The basic structure of the bundle is recalled in Section 2. In Section 3 we study the stable homotopy type and establish a stable splitting theorem as a consequence of Miller's results [5] on Stiefel manifolds. To state a special case of the theorem we introduce the notation: $L_{k}(V, W)_{+}$ for the pointed space obtained by adjoining a disjoint basepoint to $L_{k}(V, W), G_{k, l}\left(\mathbb{R}^{n}\right)$ for the flag manifold $O(n) / O(k) \times O(l) \times O(n-(k+l)$ ) (for $k, l \geqq 0, n \geqq k+l)$. We think of a point of $G_{k, l}\left(\mathbb{R}^{n}\right)$ as a pair of orthogonal subspaces of $\mathbb{R}^{n}$ of dimension $k, l$ respectively, and write $\zeta_{k}, \eta_{l}$ for the canonical $k$ - and $l$-plane bundles. Then we have:

Proposition 1.1. For $0 \leqq k \leqq n$, the space $L_{k}\left(\mathbb{R}^{n}, \mathbb{R}^{n}\right)_{+}$splits stably as a wedge of Thom spaces:

$$
\underset{0 \leqq l \leqq n-k}{ } G_{k, l}\left(\mathbb{R}^{n}\right)^{\Lambda^{2} \eta_{l} \oplus\left(\zeta_{k} \oplus \eta_{l}\right)}
$$

In Section 4 we ask when the fibre bundle $\pi$ is fibre homotopy trivial. Our answer is only partial, but includes:

Proposition 1.2. For $0<k<n$, the bundle $\pi: L_{k}\left(\mathbb{R}^{n}, \mathbb{R}^{n}\right) \rightarrow G_{k}\left(\mathbb{R}^{n}\right)$ is fibre homotopy trivial if and only if
either
(a) $(n, k)=(2,1),(4,3)$ or $(8,7)$,
or
(b) $k=1$ and $n>2$.

In case (a), but not in case (b), the bundle is trivial as smooth fibre bundle. 


\section{The fibre bundle}

To understand the structure of the bundle $\pi$, we write $\zeta$ (or sometimes $\zeta_{k}$ ) for the canonical $k$-plane bundle over $G_{k}(V)$ naturally embedded as a sub-bundle $\zeta \subseteq V$ of the trivial bundle $V$. (It is often convenient to use the same notation for a vector space $V$ and the trivial vector bundle $B \times V \rightarrow B$ over a space $B$.) Then we can identify $\pi: L_{k}(V, W) \rightarrow G_{k}(V)$ with the bundle $L_{0}(V / \zeta, W) \rightarrow G_{k}(V)$ whose fibre at a point $K \in G_{k}(V)$ is the space $L_{0}(V / K, W)$ of vector space monomorphisms $V / K \rightarrow W$. An element $f \in L_{k}(V, W)$ is identified with the induced quotient map: $V / \operatorname{ker}(f) \rightarrow W$ at $K=\operatorname{ker}(f)$.

Next we want to replace the bundle $\pi$ by a homotopy equivalent bundle with compact fibres. To that end we now assume that the vector spaces $V$ and $W$ are equipped with (positive-definite) inner products. Write $O(V, W)$ for the Stiefel manifold of isometric linear maps $V \rightarrow W$. As is well known, $O(V, W)$ is homotopy equivalent to $L_{0}(V, W)$. More precisely, we have:

Proposition 2.1. Write $\mathrm{p}(V)$ for the vector space of self-adjoint operators $V \rightarrow V$. Then there is a natural diffeomorphism:

$$
O(V, W) \times p(V) \rightarrow L_{0}(V, W)
$$

given by

$$
(f, a) \mapsto f \circ \exp (a) .
$$

For $V=W$ this is the standard factorization of an invertible matrix as a product of an orthogonal and a positive-definite matrix. The general proof is the same. Notice that (2.1) gives a natural tubular neighbourhood of the submanifold $O(V, W)$ of $L V, W$ ) and natural stable trivialization $\tau O(V, W) \oplus p(V)=L(V, W)$ of the tangent bundle.

From now on we shall abbreviate $G_{k}(V)$ to $B$ and identify $V / \zeta$ with the orthogonal complement $\zeta^{\perp}$ of $\zeta$ in $V$. Functors on vector spaces are extended to vector bundles in the usual way. Then the naturality of (2.1) gives a diffeomorphism over $B$ :

$$
L_{0}\left(\zeta^{\perp}, W\right) \rightarrow O\left(\zeta^{\perp}, W\right) \times_{B} p\left(\zeta^{\perp}\right)
$$

We write:

$$
\tilde{E}=L_{0}\left(\zeta^{\perp}, W\right), \quad E=O\left(\zeta^{\perp}, W\right) .
$$

Thus $\tilde{E} \rightarrow B$ is our original fibre bundle $\pi$, and this is fibre homotopy equivalent to the compact fibre bundle $E \rightarrow B$.

Remark 2.3. Under the diffeomorphisms (2.2) $O\left(\zeta^{\perp}, W\right)$ corresponds to the subspace of $L_{k}(V, W)$ consisting of elements $f$ whose adjoint $f^{*}$ satisfies: $f=f f^{*} f$.

Lemma 2.4. The bundle $E \rightarrow B$ admits a cross-section if and only if $\operatorname{dim} V \leqq \operatorname{dim} W$.

This is easy. We include the proof because it introduces notation required later. The 
Grassmann manifold $G_{1}(V)$ will usually be written as the projective space $P(V)$ and $\zeta_{1}$ as the Hopf bundle $H$. There are two embeddings:

$$
P\left(\mathbb{R}^{n-k+1}\right) \stackrel{i}{\rightarrow} G_{k}\left(\mathbb{R}^{n}\right) \stackrel{j}{\longleftarrow} P\left(\mathbb{R}^{k+1}\right)
$$

(for $0<k<n$ ) given as follows. A 1 -dimensional subspace $L$ of $\mathbb{R}^{n-k+1}$ is mapped by $i$ to $L \oplus \mathbb{R}^{k-1} \subseteq \mathbb{R}^{n}$, and a 1-dimensional subspace $L$ of $\mathbb{R}^{k+1}$ by $j$ to the orthogonal complement of $L \oplus \mathbb{R}^{n-k-1}$ in $\mathbb{R}^{n}$. Note that:

$$
i^{*} \zeta=H \oplus \mathbb{R}^{k-1}, \quad j^{*} \zeta^{1}=H \oplus \mathbb{R}^{n-k-1}
$$

Proof of (2.4). Take $V=\mathbb{R}^{n}$ and suppose that $E \rightarrow B$ has a section, which we can regard as a bundle monomorphism: $\zeta^{\perp} \rightarrow W$. The pull-back via $j$ is a monomorphism $H \oplus \mathbb{R}^{n-k-1} \rightarrow W$ over $P\left(\mathbb{R}^{k+1}\right)$ with orthogonal complement $\sigma$, say. Since $w_{k} \sigma \neq 0$, $\operatorname{dim} W \geqq(n-k)+k=n$.

For the remainder of the paper we shall assume that $\operatorname{dim} V \leqq \operatorname{dim} W$ and write $W$ as an orthogonal direct sum $U \oplus V$ with $\operatorname{dim} U=m, \operatorname{dim} V=n$. This splitting gives an obvious section of $E \rightarrow B$.

\section{A stable splitting}

We consider the stable homotopy type of the fibrewise one-point compactifications $\tilde{E}_{B}^{+} \rightarrow B$ and $E_{B}^{+} \rightarrow B$ obtained by adjoining a point at infinity to each fibre. (See, for example, [4].) Recall first that Miller in [5] established a stable splitting

$$
O(V, U \oplus V)^{+} \simeq \bigvee_{0 \leqq 1 \leqq n} G_{l}(V)^{\alpha\left(\zeta_{1}\right) \oplus\left(U \otimes G_{l}\right)}
$$

Here the superscript " + " again denotes one-point compactification, and $\mathfrak{o}\left(\zeta_{l}\right)$ is the vector bundle with fibre at a point $K \in G_{l}(V)$ the Lie algebra of the orthogonal group $O(K)$ of the vector space $K$. Miller's constructions can be performed equivariantly with respect to the action of the orthogonal groups $O(U)$ and $O(V)$, as in [1]. The result thus extends directly to a splitting theorem for a bundle of Stiefel manifolds by replacing $U$ and $V$ by vector bundles. We apply this to $E \rightarrow B$ to conclude:

Proposition 3.2. There is a natural stable splitting over B:

$$
E_{B}^{+} \simeq \underset{0 \leqq l \leqq n-k}{\bigvee_{B}} G_{l}\left(\zeta^{\perp}\right)_{B}^{(\eta \eta) \oplus(U \oplus \emptyset \otimes \eta}
$$

as a wedge over $B$ of Thom spaces over $B$.

For clarity we have here used $\eta_{l}$ for the canonical $l$-plane bundle over the Grassmann bundle $G_{l}\left(\zeta^{\perp}\right)$. The concept of "Thom space over $B$ " should be self-explanatory: the fibre 
over $K \in B$ is the Thom space

$$
G_{l}\left(K^{\perp}\right)^{\mathrm{o}(\eta) \oplus(U \oplus K) \otimes \eta !}
$$

By collapsing the section $B$ at infinity in $E_{B}^{+}$to a point we obtain $E_{B}^{+} / B=E^{+}$and (3.2) gives a splitting of $E^{+}$. Now $E^{+}$is homotopy equivalent to the space $L_{k}(V, U \oplus V)_{+}$ obtained by adjoining a disjoint basepoint to $\widetilde{E}$ (rather than compactifying).

Corollary 3.3. There is a natural stable splitting:

$$
L_{k}(V, U \oplus V)_{+} \simeq \bigvee_{0 \leqq 1 \leqq n-k} G_{k, 1}(V)^{\mathrm{o}(\eta) \oplus\left(U \oplus \xi_{k}\right) \otimes \eta_{1}} .
$$

As in the special case (1.1), $G_{k, l}(V)$ is the space of pairs $(K, L)$ of orthogonal subspaces of $V$ of dimension $k, l$ respectively. The case $k=0$ is Miller's original theorem.

\section{The question of triviality}

In this section we ask when the bundle $\tilde{E} \rightarrow B$ is fibre homotopy trivial, or, more restrictively, trivial as smooth fibre bundle. Notice that, if $\widetilde{E} \rightarrow B$ is fibre homotopy trivial, then so is $E \rightarrow B$, and $E_{B}^{+} \rightarrow B$ is certainly stably fibre homotopy trivial. If $\tilde{E} \rightarrow B$ is trivial in the strong sense, then so is $\tilde{E}_{B}^{+} \rightarrow B$, and both $E_{B}^{+} \rightarrow B$ and $\tilde{E}_{B}^{+} \rightarrow B$ are stably fibre homotopy trivial. It is stable triviality that we investigate first.

\section{Proposition 4.1.}

(i) If $E_{B}^{+} \rightarrow B$ is stably fibre homotopy trivial, then the sphere-bundle $\left(\mathrm{o}\left(\zeta^{\perp}\right) \oplus(U \oplus \zeta) \otimes \zeta^{\perp}\right)_{B}^{+}$is stably fibre homotopy trivial.

(ii) If $\widetilde{E}_{B}^{+} \rightarrow B$ is stably fibre homotopy trivial, then so is $\left((U \oplus V) \otimes \zeta^{\perp}\right)_{B}^{+}$.

Proof of (i). Write $F$ for a fibre of $E \rightarrow B$; it is a Stiefel manifold of dimension $N$, say. Since $F$ is connected and admits a framing, there is a stable map $e: S^{N} \rightarrow F^{+}$which induces an isomorphism of integral homology groups in dimension $N$. Writing $\xi$ for the vector bundle $\mathrm{o}\left(\zeta^{\perp}\right) \oplus(U \oplus \zeta) \otimes \zeta^{\perp}$ over $B$, let $p: E_{B}^{+} \rightarrow \xi_{B}^{+}$be the projection onto the top factor, $l=n-k$, in the decomposition (3.2).

Now suppose that we have a stable trivialization $t: B \times F^{+} \rightarrow E_{B}^{+}$over $B$. Then $p \circ t \circ(1 \times e): B \times S^{N} \rightarrow \xi_{B}^{+}$is a (stable) fibre homotopy equivalence, by Dold's lemma, since it induces a homology isomorphism in each fibre.

This completes the proof of (i). The proof of (ii) is similar, using the equivalence: $\tilde{E}_{B}^{+} \simeq E_{B}^{+} \wedge_{B}\left(\mathfrak{p}\left(\zeta^{\perp}\right)\right)_{B}^{+}$given by $(2.2)$. Observe that $\left(\mathfrak{o}\left(\zeta^{\perp}\right) \oplus(U \oplus \zeta) \oplus \zeta^{1}\right) \oplus \mathfrak{p}\left(\zeta^{1}\right)$ is isomorphic to $(U \oplus V) \oplus \zeta^{\perp}$.

Remark 4.2. It is, of course, unnecessary to use the strength of (3.2) to obtain the projection $p$ "onto the top cell" of $E$. We simply take a tubular neighbourhood (over $B$ ) of the standard section $B \rightarrow E$. The normal bundle is $\xi$, and the Pontrjagin-Thom construction gives the required projection $p$. 
Recall that $\tilde{E} \rightarrow B$ is the bundle $L_{k}(V, U \oplus V) \rightarrow G_{k}(V)$ and that $\operatorname{dim} U=m, \operatorname{dim} V=n$. We shall discuss the triviality problem under three headings: (a) $1<k<n-1$, (b) $k=n-1$, (c) $k=1$. Standard facts about vector and sphere-bundles over real projective space will be used without comment; they can be found in texts such as [2], [3].

(a) $1<k<n-1$

Proposition 4.3. If $1<k<n-1$, then $\tilde{E} \rightarrow B$ is not fibre homotopy trivial.

This is an easy corollary of (4.1) (i). Indeed, we shall show that if $E_{B}^{+} \rightarrow B$ is stably fibre homotopy trivial, $0<k<n$, then

$$
\begin{gathered}
-m-k+1 \equiv 0(\bmod a(n-k+1)) \\
m+k+1 \equiv 0(\bmod a(k+1)),
\end{gathered}
$$

where $a(r)$ is the Hurwitz-Radon number, the order of $[H]-1$ in $K O^{0}\left(P\left(\mathbb{R}^{r}\right)\right)$. The proposition then follows; for, if $k>1$ and $n-k>1$, then both $a(k+1)$ and $a(n-k+1)$ are divisible by 4 .

Proof of (4.4). As above set $\xi=\mathfrak{o}\left(\zeta^{\perp}\right) \oplus\left(\mathbb{R}^{m} \oplus \zeta\right) \otimes \zeta^{\perp}$. Then, by (4.1)(i), $\xi_{B}^{+}$is stably fibre homotopy trivial. The congruences (4.4) are just the conditions that the restriction of $\xi_{B}^{+}$to each of the subspaces $P\left(\mathbb{R}^{n-k+1}\right)$ and $P\left(\mathbb{R}^{k+1}\right)$ as in $(2.5)$ is stably fibre homotopy trivial.

We give the details in the first case; the second is similar. It is convenient to think of $\mathrm{o}\left(\zeta^{\perp}\right)$ as the exterior square $\lambda^{2}\left(\zeta^{\perp}\right)$. Then we have, by $(2.6)$,

$$
\left[i^{*} \zeta\right]=\lambda^{2}(n-k+1-[H])+(m+k-1+[H])(n-k+1-[H])
$$

in $K O^{0}\left(P\left(\mathbb{R}^{n-k+1}\right)\right)$. Using the identity $\lambda^{2}(x+y)=\lambda^{2} x+x y+\lambda^{2} y$ we obtain:

$$
\left[i^{*} \xi\right]-\operatorname{dim} \xi=(-m-k+1)([H]-1) .
$$

So the sphere-bundle associated to $i^{*} \xi$ is stably fibre homotopy trivial if and only if $a(n-k+1)$ divides $-m-k+1$.

(b) $k=n-1$

In this case, by taking orthogonal complements we can identify $B=G_{n-1}(V)$ with $G_{1}(V)=P(V)$ and $\zeta^{\perp}$ with the Hopf line bundle $H$. The bundles $\widetilde{E} \rightarrow B$ and $E \rightarrow B$ become $L_{0}(H, U \oplus V) \rightarrow P(V)$ and $O(H, U \oplus V) \rightarrow P(V)$, or, equivalently, the complement of the zero-section in the vector bundle $L H, U \oplus V) \rightarrow P(V)$ and the unit sphere bundle. If the sphere-bundle is stably fibre homotopy trivial then $a(n)$ must divide $m+n$. Conversely, if $m+n \equiv 0(\bmod a(n))$, then the vector bundle $(m+n) H$ is trivial.

Proposition 4.5. If $1<k=n-1$, then $\tilde{E} \rightarrow B$ is fibre homotopy trivial if and only if $m+n \equiv 0(\bmod a(n))$. When this condition holds, both $\widetilde{E} \rightarrow B$ and $E \rightarrow B$ are trivial as smooth bundles. 
(c) $k=1$

The final case is the most difficult. We are considering the bundles $L_{0}\left(H^{\perp}, U \oplus V\right) \rightarrow P(V)$ and $O\left(H^{\perp}, U \oplus V\right) \rightarrow P(V)$, where $H^{\perp}$ is the orthogonal complement of $H$ in $V$.

Proposition 4.6. For $k=1<n, \widetilde{E} \rightarrow B$ is trivial as bundle if and only if $n=2,4$ or 8 and $m \equiv 0(\bmod a(n))$. In these cases $E \rightarrow B$ is also trivial.

Proof. We first establish the necessity of the condition. If $\widetilde{E} \rightarrow B$ is trivial, then it is certainly fibre homotopy trivial and (4.4) gives the restriction: $m \equiv 0(\bmod a(n))$. But now both clauses (i) and (ii) of (4.1) apply, so that $\mathfrak{p}\left(H^{\perp}\right)_{B}^{+}$must be stably fibre homotopy trivial, that is: $n \equiv 0(\bmod a(n))$.

For the converse, observe that $L_{0}\left(H^{\perp}, U \oplus V\right)$ is naturally identified with $L_{0}\left(H^{\perp} \otimes H,(U \oplus V) \otimes H\right)$ by taking the tensor product with the identity on the line bundle $H$. But $H^{\perp} \otimes H$ is the tangent bundle of $P(V)$ and this is trivial if $n=2,4$ or 8 . If $m+n \equiv 0(\bmod a(n))$, then $(U \oplus V) \otimes H$ is trivial. This establishes the triviality of $\widetilde{E} \rightarrow B$, and the same argument shows that $E \rightarrow B$ is also trivial.

We complete the proof of (1.2) by verifying:

Proposition 4.7. If $k=1$ and $m=0$, then $E \rightarrow B$ is trivial as bundle.

Proof. We can give an explicit trivialization: $P(V) \times S O(V) \rightarrow O\left(H^{\perp}, V\right)$ of the bundle $E \rightarrow B$ by mapping $(K, g)$, where $K$ is a 1-dimensional subspace of $V$ and $g$ is an element of the special orthogonal group of $V$, to the composition $K^{\perp} \subseteq V \rightarrow V$ of $g$ with the inclusion.

It remains to examine the question of the triviality or fibre homotopy triviality of $E \rightarrow B$ for $k=1$ and $m>0$. We have been unable to give an answer even in the first interesting case $n=3$. Our present knowledge is collected in the final proposition. Part (i) is based on a suggestion of D. Hacon.

Proposition 4.8. For $k=1, m>0, n=3$ :

(i) the bundle $E \rightarrow B$ is trivial if $m=4$;

(ii) the bundle $E \rightarrow B$ is not fibre homotopy trivial if $m+4$ is not a power of 2;

(iii) the bundle $E_{B}^{+} \rightarrow B$ is stably fibre homotopy trivial if and only if $m \equiv 0(\bmod 4)$.

Proof of (i). We take $U$ to be the space $\mathbb{t}$ of quaternions, $V$ the space of pure quaternions, and regard $U \oplus V$ (or $V \oplus U$ ) as the space of pure Cayley numbers. Then the group $G_{2}$ of automorphisms of the Cayley numbers acts orthogonally on $U \oplus V$. The action is transitive on the Stiefel manifold of orthonormal 2-frames in $U \oplus V$, and the stabilizer of any 2-frame in $V$ is the subgroup $S p(1)$ which fixes the whole of $V$.

We obtain an explicit trivialization: $P(V) \times G_{2} / S p(1) \rightarrow O\left(H^{\perp}, U \oplus V\right)$, along the lines of (4.7), by sending $(K, g S p(1))$ to the composition $K^{\perp} \subseteq V \subseteq U \oplus V \rightarrow U \oplus V$ of $g$ and the inclusion. 
Proof of (ii). Suppose that the bundle $O\left(H^{1}, \mathbb{R}^{m+3}\right) \rightarrow P\left(\mathbb{R}^{3}\right)$ is fibre homotopy trivial. Then so is its restriction to the subspace $P\left(\mathbb{R}^{2}\right)$. This restricted bundle can be identified with $O\left(\mathbb{R} \oplus H, \mathbb{R}^{m+3}\right) \rightarrow P\left(\mathbb{R}^{2}\right)$, because $2 H$ is trivial, or with the mapping torus of the involution $T$ of the Stiefel manifold of 2-frames in $\mathbb{R}^{m+3}$ which changes the sign of the second vector. Since the bundle is fibre homotopy trivial, $T$ must be homotopic to the identity. The condition on $m$ now follows from a theorem of James $([3,(23.10)]$. (The same theorem implies that $E \rightarrow B$ is never fibre homotopy trivial in the cases $k=1, m>0$, $n=5$ or 9 .)

Proof of (iii). We show that each of the components in the decomposition (3.2) is stably trivial if $4 \mid m$. Only the middle term $P\left(H^{\perp}\right)_{B}^{(U \oplus H) \otimes \eta}$, where $\eta$ is the Hopf line bundle over the projective bundle $P\left(H^{\perp}\right)$, causes difficulty. Observe first that $P\left(H^{\perp}\right)$ can be embedded in the trivial bundle $B \times P(V)$ by inclusion of $H^{\perp}$ in $V$; the bundle $U \otimes \eta$ over $P\left(H^{\perp}\right)$ is the restriction of the bundle $U \otimes H$ over $B \times P(V)$, and this is trivial because $m$ is divisible by $a(n)$. So we must show that $P\left(H^{\perp}\right)_{B}^{H \otimes n}$ is stably trivial.

Now the cofibre sequence over $B$ :

$$
P(H)_{B}^{+} \rightarrow P\left(H^{\perp} \oplus H\right)_{B}^{+} \rightarrow P\left(H^{\perp}\right)_{B}^{H \otimes \eta},
$$

given by the inclusion of $H$ in $H^{\perp} \oplus H$, is split by the projection $P\left(H^{\perp} \oplus H\right) \rightarrow B=P(H)$. But the bundle $P\left(H^{\perp} \oplus H\right)_{B}^{+}$is trivial, since $H^{\perp} \oplus H=V$. By splitting the trivial bundle we obtain a stable equivalence:

$$
P\left(H^{\perp}\right)_{B}^{H \otimes n} \vee_{B}\left(B \times S^{0}\right) \underset{B}{\simeq}\left(B \times P\left(\mathbb{R}^{n-1}\right)^{H}\right) \vee_{B}\left(B \times S^{0}\right)
$$

Inclusion and projection gives a stable map $P\left(H^{\perp}\right)_{B}^{H \otimes n} \rightarrow B \times P\left(\mathbb{R}^{n-1}\right)^{H}$ which is an equivalence on fibres and hence a stable fibre homotopy equivalence. (Alternatively, decompose both sides of the equivalence:

$$
\left.O\left(H^{\perp}, \mathbb{R}^{3}\right) \simeq B \times O\left(\mathbb{R}^{2}, \mathbb{R}^{3}\right),(4.7) .\right)
$$

Acknowledgements. We owe one result in Section 4 to Derek Hacon, and it is a pleasure to record out thanks to him. The second author is grateful to the S.E.R.C. for a Visiting Fellowship under grant G.R/D/313324 at the University of Aberdeen.

\section{REFERENCES}

1. M. C. СRABB, On the stable splitting of $U(n)$ and $\Omega U(n)$, in Algebraic Topology Barcelona 1986 (Springer Lecture Notes in Math. 1298, 1987), 35-53.

2. D. Husemoller, Fibre Bundles (McGraw-Hill, New York, 1966).

3. I. M. James, The Topology of Stiefel Manifolds (Cambridge University Press, 1976).

4. I. M. JAMEs, General Topology and Homotopy Theory (Springer-Verlag, New York, 1984).

5. H. Miller, Stable splittings of Stiefel manifolds, Topology 24 (1985), 411-419.

Department of Mathematics

UNIVERSITY OF ABERDEEN

AbERDEEN AB9 2TY

SCOTLAND
Instituto de Matemática e Estatistica Universidade de Sao Paulo

Sáo Paulo

Brazil 